\title{
Intracule functional models: I. Angle-corrected correlation kernels
}

\author{
Elise E. Dumont, $\dagger$ Deborah L. Crittenden $\ddagger$ and Peter M. W. Gill*
}

Received 22nd June 2007, Accepted 23rd July 2007

First published as an Advance Article on the web 13th August 2007

DOI: $10.1039 / b 709513 a$

We explore the merits of applying a simple angle-dependent correction to the correlation kernel within the framework of Hartree-Fock-Wigner theory. Based on numerical results for the first eighteen atoms, we conclude that such a correction offers a significant improvement over the action kernel that we and others have explored previously.

\section{Introduction}

Hartree-Fock (HF) theory ${ }^{1-3}$ often yields fairly accurate predictions of molecular structure but it is much less satisfactory for many other properties. In particular, its simplistic treatment of electron motion fails to account properly for the formation of an electron pair during bond formation and it has been known for many years that quantitative predictions are possible only if the theoretical model is extended to account for electron correlation. Allowing the electrons to avoid one another stabilizes the system and the difference between its exact and HF energies is known ${ }^{4,5}$ as the correlation energy $E_{\mathrm{c}}$. The difficulty of calculating $E_{\mathrm{c}}$ is known as "the correlation problem" and has been the most challenging obstacle to the progress of quantum chemistry during its eighty-year history.

A few years ago, Rassolov argued ${ }^{6}$ that the strength of the correlation of two electrons depends on both their separation $\boldsymbol{u}=\boldsymbol{r}_{1}-\boldsymbol{r}_{2}$ in position space and their separation $\boldsymbol{v}=\boldsymbol{p}_{1}-\boldsymbol{p}_{2}$ in momentum space. We believe that this insight is a profound one but, because the Heisenberg uncertainty principle precludes the construction of a phase-space wavefunction, we have turned instead to Wigner's ${ }^{7,8}$ reduced second-order phase-space distribution $W\left(\boldsymbol{r}_{1}, \boldsymbol{r}_{2}, \boldsymbol{p}_{1}, \boldsymbol{p}_{2}\right)$ to provide a relatively simple function, the Omega intracule $\Omega(u, v, \omega)$, that gives the joint quasi-probability density of $u, v$ and $\omega$, the last of these being the angle between $\boldsymbol{u}$ and $\boldsymbol{v}^{9,10}$ Besley has argued ${ }^{11}$ that it may be preferable to derive phase-space intracules from the rigorously non-negative Husimi distribution ${ }^{12}$ but we do not explore this possibility here.

We have suggested that the correlation energy of a system can be found by contracting its HF Omega intracule with a suitable correlation kernel $G(u, v, \omega)$. Using arguments based on the known correlation energies of the helium-like ions, we have assumed in our work to date that the correlation kernel depends on the product $s=u v$ but is independent of $\omega$. A number of investigations ${ }^{9,13-16}$ of this assumption have been published and they have concluded that, although such kernels can provide surprisingly good estimates of ground-state atom-

Research School of Chemistry, Australian National University, ACT 0200 Australia.E-mail: peter.gill@anu.edu.au

$\dagger$ EED acknowledges postdoctoral support from ARC grant DP0664466.

$\ddagger$ DLC acknowledges postdoctoral support from ARC grant DP0771978. ic correlation energies, they seem to be less effective in describing the variations of $E_{\mathrm{c}}$ across isoelectronic molecules.

To obtain even higher accuracy, it is clear that more flexible kernels are needed. In this paper, we explore the possibility of adding a small $\omega$-based correction and present results for a number of atoms. We define the correlation energy to be the difference between the complete-basis UHF energy and the exact eigenvalue of the non-relativistic Schrödinger equation and we use atomic units throughout.

\section{Angle intracules}

In calculations using one-electron basis functions $\phi_{a}(\boldsymbol{r})$, the Omega intracule is ${ }^{10}$

$$
\Omega(u, v, \omega)=\sum_{a b c d} \Gamma_{a b c d}[a b c d]_{\Omega}
$$

where $\Gamma_{a b c d}$ is a two-particle density matrix element and the Omega integrals

$$
[a b c d]_{\Omega}=\int \Phi_{a d}^{*}(\boldsymbol{u}, \boldsymbol{v}) \Phi_{b c}(\boldsymbol{u}, \boldsymbol{v}) \delta\left(\theta_{u v}-\omega\right) \mathrm{d} \boldsymbol{\Omega}_{\boldsymbol{u}} \mathrm{d} \boldsymbol{\Omega}_{\boldsymbol{v}}
$$

are formed from the phase functions

$$
\Phi_{a d}(\boldsymbol{u}, \boldsymbol{v})=(2 \pi)^{-3 / 2} \int \phi_{a}(\boldsymbol{r}) \phi_{d}(\boldsymbol{r}+\boldsymbol{u}) e^{i \boldsymbol{v} \cdot \boldsymbol{r}} \mathrm{d} \boldsymbol{r}
$$

The Omega integrals are more difficult than the analogous $[a b \mid c d]$ Coulomb integrals but we have shown how they can be calculated efficiently over Gaussian basis functions, exploiting Boys' approach ${ }^{17}$ in which the fundamental $[s s s s]_{\Omega}$ integral is found and differentiated with respect to the coordinates of the Gaussians to yield $[a b c d]_{\Omega}$ of higher angular momentum.

We have reported intracules for various atoms and molecules, in ground and excited states (see ref. 9 and references therein) and, most recently, we have studied the Angle intracule

$$
\Upsilon(\omega)=\int_{0}^{\infty} \int_{0}^{\infty} \Omega(u, v, \omega) \mathrm{d} u \mathrm{~d} v
$$

which gives the (quasi-)probability density of $\omega$. In a system where the directions of $\boldsymbol{u}$ and $\boldsymbol{v}$ are statistically independent, such as two identical harmonic oscillators, ${ }^{18}$ the Angle intracule is determined entirely by the appropriate geometric 
Table 1 Fourier coefficients, exact correlation energies, LYP errors and kernel-based errors ${ }^{a-f}$

\begin{tabular}{|c|c|c|c|c|c|c|c|c|c|}
\hline & $\frac{n(n-1)}{4}$ & $d_{1}$ & $-d_{3}$ & $d_{5}$ & $-d_{7}$ & $E_{\mathrm{c}}$ & $\Delta E_{\mathrm{c}}^{\mathrm{LYP}}$ & $\Delta E_{\mathrm{c}}^{2}$ & $\Delta E_{\mathrm{c}}^{3}$ \\
\hline$C_{s}$ & & & & & & & & 0.1060 & 0.1008 \\
\hline$C_{\omega}^{s}$ & & & & & & & & 0 & 0.0075 \\
\hline$\zeta$ & & & & & & & & 0.9163 & 0.9101 \\
\hline $\mathrm{H}$ & 0.0 & 0.0000 & 0.0000 & 0.0000 & 0.0000 & 0.0 & 0.0 & 0.0 & 0.0 \\
\hline $\mathrm{He}$ & 0.5 & 0.0237 & 0.0804 & 0.0183 & 0.0054 & 42.1 & 1.7 & 1.3 & 0.5 \\
\hline $\mathrm{Li}$ & 1.5 & 0.0213 & 0.0698 & 0.0114 & 0.0029 & 45.4 & 8.1 & 2.3 & 1.3 \\
\hline $\mathrm{Be}$ & 3.0 & 0.0194 & 0.0630 & 0.0125 & 0.0104 & 94.4 & 0.2 & -3.3 & -5.9 \\
\hline B & 5.0 & 0.0466 & 0.1447 & 0.0112 & 0.0072 & 120.8 & 5.5 & 0.2 & -2.2 \\
\hline $\mathrm{C}$ & 7.5 & 0.0950 & 0.3019 & 0.0211 & 0.0057 & 151.3 & 8.2 & 3.3 & 2.2 \\
\hline $\mathrm{N}$ & 10.5 & 0.1723 & 0.5412 & 0.0440 & 0.0065 & 184.7 & 7.5 & 7.3 & 8.6 \\
\hline $\mathrm{O}$ & 14.0 & 0.2495 & 0.7868 & 0.0682 & 0.0070 & 248.5 & 9.8 & 2.5 & 5.2 \\
\hline $\mathrm{F}$ & 18.0 & 0.3408 & 1.0763 & 0.0955 & 0.0082 & 317.8 & 4.4 & -4.3 & 0.5 \\
\hline $\mathrm{Ne}$ & 22.5 & 0.4657 & 1.4792 & 0.1474 & 0.0153 & 390.8 & -7.2 & -11.3 & -3.0 \\
\hline $\mathrm{Na}$ & 27.5 & 0.4199 & 1.3141 & 0.0927 & 0.0018 & 395.9 & 12.5 & -7.8 & -1.5 \\
\hline $\mathrm{Mg}$ & 33.0 & 0.3644 & 1.1269 & 0.0591 & 0.0066 & 438.4 & 21.1 & -3.3 & -0.7 \\
\hline $\mathrm{Al}$ & 39.0 & 0.3341 & 1.0226 & 0.0379 & 0.0104 & 465.2 & 29.6 & 0.8 & 1.5 \\
\hline $\mathrm{Si}$ & 45.5 & 0.3303 & 1.0046 & 0.0281 & 0.0143 & 500.2 & 30.6 & -2.5 & -2.5 \\
\hline $\mathrm{P}$ & 52.5 & 0.3195 & 0.9631 & 0.0191 & 0.0281 & 539.8 & 26.5 & -1.9 & -2.8 \\
\hline S & 60.0 & 0.3216 & 0.9701 & 0.0277 & 0.0431 & 596.8 & 33.1 & 1.5 & -0.8 \\
\hline $\mathrm{Cl}$ & 68.0 & 0.3200 & 0.9652 & 0.0361 & 0.0621 & 658.3 & 33.0 & 4.1 & 0.4 \\
\hline $\mathrm{Ar}$ & 76.5 & 0.3139 & 0.9434 & 0.0400 & 0.0826 & 722.7 & 28.1 & 7.6 & 2.3 \\
\hline RMSD & & & & & & & 19.0 & 4.7 & 3.2 \\
\hline MAD & & & & & & & 14.8 & 3.6 & 2.3 \\
\hline
\end{tabular}

${ }^{a}$ All energies in millihartrees. Correlation energies are taken to be positive numbers. ${ }^{b} E_{\mathrm{c}}=$ exact value taken from ref. $21 .{ }^{c} \Delta E_{\mathrm{c}}^{\mathrm{LYP}}=$ error of the LYP density functional. ${ }^{d} \Delta E_{\mathrm{c}}^{2}=$ error of the two-parameter kernel, eqn (3.15). ${ }^{e} \Delta E_{\mathrm{c}}^{3}=$ error of the three-parameter kernel, eqn (3.17).

${ }^{f}$ LYP and kernel calculations used UHF/6-311G wavefunctions.

Jacobian and it is easy to show that

$$
\Upsilon_{0}(\omega)=\frac{n(n-1)}{2} \frac{\sin \omega}{2}
$$

In more interesting systems, it is useful to take this as a baseline and write

$$
\Upsilon(\omega)=\Upsilon_{0}(\omega)+\Delta \Upsilon(\omega)
$$

Because both $\Upsilon(\omega)$ and $\Upsilon_{0}(\omega)$ are normalized to $n(n-1) / 2$, the differential intracule $\Delta \Upsilon(\omega)$ has no net content. However, its subtle features provide a lens through which one can perceive important changes in the mutual orbital motion of electrons.

For example, we found that $\Delta \Upsilon(\omega)$ for a helium atom is positive around $\omega=\pi / 2$ and correspondingly negative around $\omega=0$ and $\omega=\pi$. This is significant because, at $\omega=\pi / 2$, the electrons' relative momentum vector is perpendicular to their relative position vector, indicating a circular orbit. In the $\mathrm{H}_{2}$ molecule, $\Delta \Upsilon(\omega)$ is also positive in this region, but less so than in the helium atom, indicating that the tendency for the electrons to orbit one another is weaker in the molecule than in the atom. Moreover, as the molecule is stretched, $\Delta \Upsilon(\omega)$ flattens even further until, in the dissociated limit, it vanishes completely. (See Fig. 4 in ref. 10.)

A similar trend is observed as a neon atom is transmuted successively into $\mathrm{HF}, \mathrm{H}_{2} \mathrm{O}, \mathrm{NH}_{3}$ and $\mathrm{CH}_{4}$, indicating that the localization of the neon lone-pair electrons into the $\sigma$ bonds of the molecules increases the ellipticity of their orbits. (See Fig. 8 in ref. 10)
It is convenient to characterize the differential intracule via its Fourier expansion

$$
\begin{gathered}
\Delta \Upsilon(\omega)=d_{1} \sin \omega+d_{3} \sin 3 \omega+d_{5} \sin 5 \omega+\ldots \\
d_{k}=\frac{2}{\pi} \int_{0}^{\pi} \Delta \Upsilon(\omega) \sin k \omega \mathrm{d} \omega
\end{gathered}
$$

and we note that the integration of (2.7) yields the sum rule

$$
0=d_{1}+\frac{d_{3}}{3}+\frac{d_{5}}{5}+\ldots
$$

We have evaluated $d_{1}, d_{3}, d_{5}$ and $d_{7}$ for the UHF/6-311G wavefunctions of the first 18 atoms in the periodic table and they are listed in columns 3-6 of Table 1 . By comparing the $d_{k}$ with $n(n-1) / 4$, we see that $\Delta \Upsilon(\omega)$ is never more than a small component of $\Upsilon(\omega)$ and that its contribution falls from roughly $20 \%$ in the helium atom, to $10 \%$ in neon, and to less than $2 \%$ in argon. Further examination reveals that the Fourier expansion (2.7) seems to converge rapidly and $d_{3}$ is always the largest coefficient. Moreover, we find $d_{3} \approx-3 d_{1}$ in all cases, as the sum rule (2.9) would predict for a rapidly converging series. From these observations, we conclude that the $d_{3}$ values capture most of the information in the $\Delta \Upsilon(\omega)$.

The $d_{3}$ values are plotted as a function of the atomic number $Z$ in Fig. 1 and it is immediately clear that they reflect the atomic shell structure. The detailed variations can be rationalized by recognizing that $d_{3}$ is a sum of contributions from all $n(n-1) / 2$ pairs of electrons in the atom and that, whereas two electrons in the same shell give a negative contribution, two electrons in different shells give a smaller positive contribution. $^{10}$ 


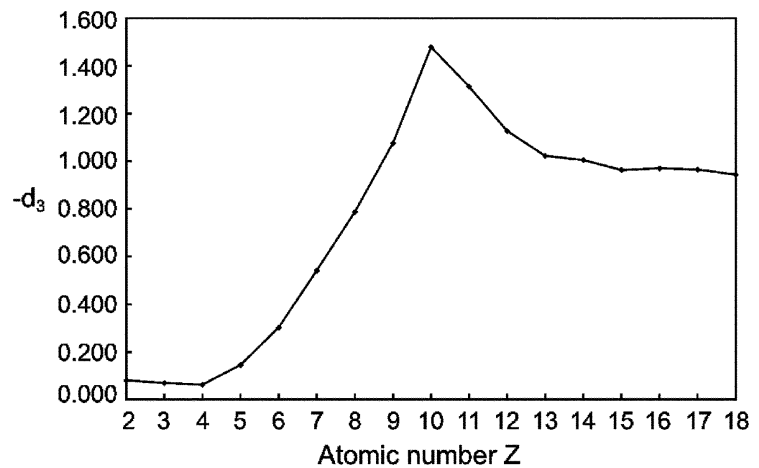

Fig. 1 The $d_{3}$ Fourier coefficients for the first 18 atoms. Based on $\mathrm{UHF} / 6-311 \mathrm{G}$ wavefunctions.

The $d_{3}$ value in helium is -0.0804 and, as described above, this reflects the fact that the two electrons are often found orbiting each other. When a third electron is added (Li), it goes into the $2 \mathrm{~s}$ subshell. Through its interaction with the two 1s electrons, this electron introduces two new positive contributions and, as a result, $d_{3}$ becomes slightly smaller. The addition of a fourth electron $(\mathrm{Be})$ creates two positive contributions and one larger negative contribution and the value of $d_{3}$ remains almost unchanged. However, as the next six electrons (B to $\mathrm{Ne}$ ) are successively added to the $2 p$ subshell, the additional negative contributions significantly outweigh the positive contributions and $d_{3}$ grows quadratically.

As we move to sodium, an electron is added to the $3 \mathrm{~s}$ subshell, leading to ten new positive contributions and a precipitous drop in $d_{3}$. The addition of the twelfth electron $(\mathrm{Mg})$ has a similar effect but the subsequent six additions ( $\mathrm{Al}$ to Ar) reduce $d_{3}$ much less, as the new positive and negative contributions almost balance.

\section{Intracule-based correlation models}

We have conjectured ${ }^{9}$ that an Omega correlation energy functional exists, i.e.

$$
E_{\mathrm{c}}=F[\Omega(u, v, \omega)]
$$

and we have additionally speculated that it can be written

$$
E_{\mathrm{c}}=\int_{0}^{\infty} \int_{0}^{\infty} \int_{0}^{\pi} \Omega(u, v, \omega) G(u, v, \omega) \mathrm{d} \omega \mathrm{d} v \mathrm{~d} u
$$

where $G(u, v, \omega)$ is a universal correlation kernel. Substituting eqn (2.1) into eqn (3.11) yields

$$
E_{\mathrm{c}}=\sum_{a b c d} \Gamma_{a b c d}[a b c d]_{\mathrm{G}}
$$

where we have introduced the correlation integral

$$
[a b c d]_{\mathrm{G}}=\int \Phi_{a d}^{*}(\boldsymbol{u}, \boldsymbol{v}) \Phi_{b c}(\boldsymbol{u}, \boldsymbol{v}) G(u, v, \omega) \mathrm{d} u \mathrm{~d} v
$$

In the case where each of the basis functions is an s-type Gaussian, this becomes

$$
\begin{aligned}
{[s s s]_{\mathrm{G}}=} & \frac{1}{[4(\alpha+\delta)(\beta+\gamma)]^{3 / 2}} \iint e^{-\lambda^{2} u^{2}-\mu^{2} v^{2}-i \eta \boldsymbol{u} \cdot \boldsymbol{v}-\boldsymbol{P} \cdot \boldsymbol{u}-i \boldsymbol{Q} \cdot \boldsymbol{v}-R} \\
& \times G(u, v, \omega) \mathrm{d} \boldsymbol{u} \mathrm{d} \boldsymbol{v}
\end{aligned}
$$

and the various constants depend on the Gaussian exponents and centers. ${ }^{9}$

We and others have explored the two-parameter correlation kernel

$$
G(u, v, \omega)=C_{s} j_{0}(\zeta s)
$$

where $s=u v$, and this produces the concentric fundamental integral

$$
\begin{aligned}
& {[s s s s]_{j_{0}}=\frac{\pi^{3}}{[(\alpha+\delta)(\beta+\gamma)]^{3 / 2}}} \\
& \frac{\left[4 \lambda^{2} \mu^{2}+(\zeta-\eta)^{2}\right]^{-1 / 2}-\left[4 \lambda^{2} \mu^{2}+(\zeta+\eta)^{2}\right]^{-1 / 2}}{2 \zeta \eta}
\end{aligned}
$$

However, in the light of the discussion in the preceding section, we expect that the correlation energy of a pair of electrons will be influenced by the ellipticity of their orbit, and we are now led to consider the three-parameter angle-corrected correlation kernel

$$
G(u, v, \omega)=C_{s} j_{0}(\zeta s)+C_{\omega} \sin 3 \omega
$$

The term $\sin (2 k+1) \omega$ gives rise to the concentric fundamental integral

$$
\begin{aligned}
& {[s s s]_{\sin }=\frac{\pi^{3}\left(1+z^{2}\right)^{3 / 2}\left(-z^{2}\right)^{k}}{[(\alpha+\beta)(\gamma+\delta)]^{3 / 2}} \frac{\Gamma\left(k+\frac{3}{2}\right)^{2}}{(2 k) !}} \\
& F\left(k+\frac{3}{2}, k+\frac{3}{2}, 2 k+2,-z^{2}\right)
\end{aligned}
$$

where $z=\eta /(2 \lambda \mu)$ and $F(a, b, c, x)$ is the hypergeometric function. ${ }^{19}$ Concentric integrals of higher angular momentum are similar to (3.16) and (3.18) but contain several such terms. Because these correlation integrals have only four-fold permutational symmetry, ${ }^{20}$ the cost of computing each set of them is approximately double that of the two-electron repulsion integrals required for the preceding HF calculation. Thus, the total cost of computing the HFW correlation energy using the two-parameter kernel is roughly equivalent to two SCF iterations, and the total cost of computing the HFW correlation energy using the three-parameter kernel is similar to performing four SCF iterations.

We have optimized the values of $C_{s}, C_{\omega}$ and $\zeta$ by a leastsquares fit to the exact unrestricted correlation energies ${ }^{21}$ of the first 18 atoms in the periodic table. In Table 1, we report both the root-mean-square deviation (RMSD), which is the quantity that was minimized, and the mean absolute deviation (MAD). All of our intracule functional calculations are based on $\mathrm{UHF} / 6-311 \mathrm{G}$ wavefunctions.

The optimized values of $C_{s}$ and $\zeta$ in the two-parameter kernel (3.15) are 0.1060 and 0.9163 , respectively. When the additional $\sin 3 \omega$ term is included, the coefficient $C_{s}$ diminishes by $5 \%$ but $\zeta$, which is more robust, ${ }^{9}$ changes by less than $1 \%$.

Table 1 compares the exact correlation energies with the estimates obtained using the popular LYP density functional, ${ }^{22}$ the two-parameter kernel and the three-parameter kernel. The errors $\Delta E_{\mathrm{c}}^{2}$ and $\Delta E_{\mathrm{c}}^{3}$ from the two kernels are shown in Fig. 2. The inclusion of the $\sin 3 \omega$ term reduces the mean absolute deviation by $36 \%$, from 3.6 to $2.3 \mathrm{~m} E_{\mathrm{h}}$, but its 


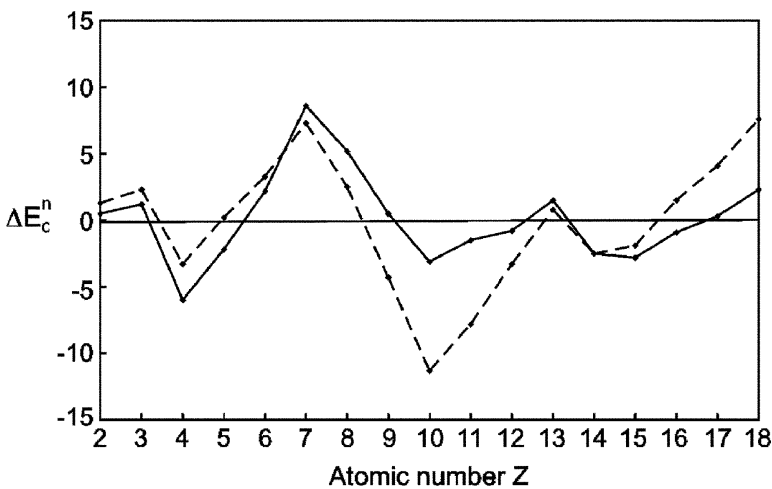

Fig. 2 Correlation energies errors $\left(\mathrm{m} E_{\mathrm{h}}\right)$ for the first 18 atoms from the two-parameter kernel (3.15) (dashed line) and three-parameter kernel (3.17) (solid line). Based on UHF/6-311G wavefunctions.

benefits are felt primarily by the heavier atoms ( $\mathrm{F}$ to Ar) whose MAD is reduced by almost a factor of three. Both kernels are much more accurate than the LYP functional and we have found that other commonly used correlation functionals (such as PW91) perform even worse.

Although the three-parameter kernel is significantly more accurate than its two-parameter predecessor, there is still room for improvement. The largest error $\left(8.6 \mathrm{~m} E_{\mathrm{h}}\right)$ occurs for the nitrogen atom, which is also the most spin-polarized system in our set, and we are encouraged by the fact that the errors across the $2 \mathrm{p}$ block (i.e. from $\mathrm{Be}$ to $\mathrm{Ne}$ ) appear to be very systematic. We are currently investigating the reason for this.

\section{Concluding remarks}

In this paper, we have sought to improve the accuracy of intracule-based electron correlation treatments through the explicit inclusion of a term that depends on the angle $\omega$ between the interelectronic position and momentum vectors, $\boldsymbol{u}$ and $\boldsymbol{v}$. We have argued that extracting the $\sin 3 \omega$ component of the intracule captures the majority of the chemically relevant correlation between $u$ and $v$ and we have explored the merits of using this as a small additive correction to the usual action-based scheme. In tests on the first eighteen atoms in the periodic table, we have shown that this correction can be applied through the introduction of a single empirical parameter and that this leads to an accuracy improvement of more than $30 \%$.

\section{References}

1 D. R. Hartree, Proc. Cambridge Philos. Soc., 1928, 24, 89.

2 J. C. Slater, Phys. Rev., 1929, 34(10), 1293-1322.

3 V. Fock, Z. Phys., 1930, 61, 126.

4 E. Wigner, Phys. Rev., 1934, 46, 1002-1011.

5 P.-O. Löwdin, Adv. Chem. Phys., 1959, 2, 207-322.

6 V. A. Rassolov, J. Chem. Phys., 1999, 110(8), 3672-3677.

7 E. Wigner, Phys. Rev., 1932, 40, 749-759.

8 H. J. Groenewold, Physica, 1946, 12, 405-460.

9 P. M. W. Gill, D. L. Crittenden, D. P. O'Neill and N. A. Besley, Phys. Chem. Chem. Phys., 2006, 8, 15-25.

10 D. L. Crittenden and P. M. W. Gill, J. Chem. Phys., 2007, 127(1), 014101.

11 N. A. Besley, Chem. Phys. Lett., 2005, 409(1-3), 63-69.

12 K. Husimi, Proc. Phys. Math. Soc. Jpn., 1940, 22, 264.

13 P. M. W. Gill and D. P. O'Neill, J. Chem. Phys., 2005, 122(9), 094110.

14 R. Fondermann, M. Hanrath, M. Dolg and D. P. O'Neill, Chem. Phys. Lett., 2005, 413(1-3), 237-241.

15 N. A. Besley, J. Chem. Phys., 2006, 125(7), 074104.

16 D. P. O'Neill and P. M. W. Gill, Recent Advances in Electron Correlation Methodology, ACS Symposium, Oxford University Press, Washington, DC, 2007.

17 S. F. Boys, Proc. R. Soc. London, Ser. A, 1950, A200, 542-554.

18 P. M. W. Gill, D. P. O'Neill and N. A. Besley, Theor. Chem. Acc., 2003, 109(5), 241-250.

19 M. Abramowitz and I. E. Stegun, Handbook of Mathematical Functions, Dover, New York, 1972.

20 N. A. Besley, D. P. O'Neill and P. M. W. Gill, J. Chem. Phys., 2003, 118(5), 2033-2038.

21 D. P. O'Neill and P. M. W. Gill, Mol. Phys., 2005, 103(6-8), 763-766.

22 C. Lee, W. Yang and R. G. Parr, Phys. Rev. B: Condens. Matter Mater. Phys., 1988, 37(2), 785-789. 